\title{
Catherine Fino, L'hospitalité, figure sociale de la charité. Deux fondations hospitalières à Québec
}

Paris, Desclée de Brouwer, coll. « Théologie à l'université », 2010, 459 p.

Katrin Langewiesche

\section{(2) OpenEdition}

\section{Journals}

Édition électronique

URL : http://journals.openedition.org/assr/23960

DOI : $10.4000 /$ assr.23960

ISSN : $1777-5825$

Éditeur

Éditions de l'EHESS

Édition imprimée

Date de publication : 30 décembre 2012

Pagination : 173

ISSN : 0335-5985

Référence électronique

Katrin Langewiesche, «Catherine Fino, L'hospitalité, figure sociale de la charité. Deux fondations hospitalières à Québec », Archives de sciences sociales des religions [En ligne], 160 | octobre-décembre 2012, mis en ligne le 11 mars 2013, consulté le 21 septembre 2020. URL : http:// journals.openedition.org/assr/23960 ; DOI : https://doi.org/10.4000/assr.23960

Ce document a été généré automatiquement le 21 septembre 2020.

(c) Archives de sciences sociales des religions 


\section{Catherine Fino, L'hospitalité, figure sociale de la charité. Deux fondations hospitalières à Québec}

Paris, Desclée de Brouwer, coll. « Théologie à l'université », 2010, 459 p.

Katrin Langewiesche

\section{RÉFÉRENCE}

Catherine Fino, L'hospitalité, figure sociale de la charité. Deux fondations hospitalières à Québec, Paris, Desclée de Brouwer, coll. « Théologie à l'université », 2010, 459 p. 
1 Catherine Fino explore les pratiques de religieuses hospitalières pour montrer la dimension sociopolitique de la charité chrétienne et son influence sur l'espace public. Cette étude sur deux grandes institutions hospitalières de la ville de Québec, l'Hôtel-Dieu de Sillery-Québec des Augustines (étudié de 1639 à 1759) et l'Asile Saint-Michel de Beauport des sœurs de la Charité de Québec (étudié de 1893 à 1939), critique la thèse de l'extinction de la charité hospitalière en relation avec l'avènement de l'hôpital moderne. Celle-ci soutient la disparition de la charité dans le contexte contemporain depuis l'essor d'une médecine de plus en plus technique, la laïcisation de la direction et du personnel des institutions de soins et la professionnalisation des métiers

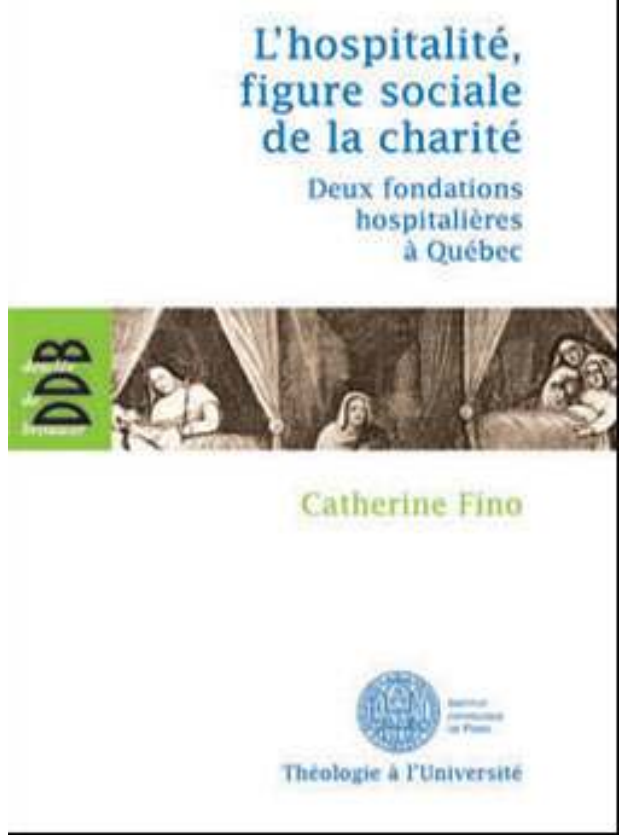
infirmiers. Michel Foucault est un des représentants célèbres de cette lecture historiographique. Catherine Fino remet en question cette manière d'écrire l'histoire qui, selon elle, ne souligne pas assez les effets sociaux et politiques des actions des religieuses. Tout en affirmant qu'évidemment l'engagement caritatif et humanitaire n'est pas réservé aux chrétiens, l'auteur explore le lien qui existe entre la foi, l'expérience spirituelle chrétienne, et la charité en acte, et met l'accent sur l'importance évangélique de l'hospitalité.

2 Catherine Fino, sœur salésienne de Bosco, médecin et théologienne, puise son argumentation dans deux disciplines académiques: l'histoire et la théologie morale. Son livre est le résultat d'une thèse de doctorat soutenue à la Faculté de Théologie et de Sciences religieuses de l'Institut catholique de Paris. Cependant, son parti-pris du départ d'une inscription sociale de la charité dans l'histoire l'éloigne de la position de certains théologiens pour qui la charité oriente l'intention du croyant sans participer à la détermination des normes.

3 L'analyse de Catherine Fino repose sur le concept de "figure sociale de la charité » qu'elle explore au cours du premier chapitre. La notion de figure sociale lui permet d'analyser les articulations entre le collectif et l'individuel, d'envisager la charité à la fois comme vertu personnelle et collective, d'étudier les actions personnelles de quelques concernées et l'action du corps institutionnel, enfin de réfléchir sur les liens entre pratiques et représentations. L'histoire des pratiques hospitalières est donc caractérisée par différentes «figures de la charité » que l'auteur décrit dans les trois chapitres suivants. Au cours des chapitres, elle montrera que l'engagement des sujets dans l'expérience de la foi et de la charité les transforme et les modèle, mais qu'il a également un impact sur les institutions et les pratiques qu'ils ont contribué à mettre en place. Elle base ses recherches sur un corpus documentaire de deux congrégations religieuses selon une périodisation qui permet, en fonction de sa problématique, de 
distinguer et de comparer trois figures sociales de la charité : le projet missionnaire caritatif au service de la conversion des Indiens (1639-1644), le projet hospitalier au service de la colonie porté par les Augustines de la Miséricorde de Jésus (1644-1759) et le projet de réforme asilaire engagé par les sœurs de la Charité de Québec (1893-1939). Les deux premiers rendent compte d'une hospitalité fondée sur une perspective religieuse tandis que le troisième étudie la mise en pratique de la charité lors du développement de la médicalisation hospitalière dans la première moitié $\mathrm{du} \mathrm{xx}^{\mathrm{e}}$ siècle.

4 Le deuxième chapitre décrit le projet des fondatrices de l'Hôtel-Dieu de Sillery qui inscrivent la charité hospitalière au service de l'évangélisation. Les Augustines se basent sur une spiritualité française du début du xviie siècle, décrite dans des ouvrages de dévotion qui font de la vie active un tremplin pour la sainteté, à parité avec la vie contemplative. Les religieuses hospitalières acceptent la maladie comme un don de Dieu et consentent au martyre de charité en s'exposant à la contagion. Dans cette optique, la petite communauté d'environ six sœurs augustines se met au service des Indiens. Elles prennent en charge leurs malades et accueillent tous ceux, infirmes et vieillards, qui ne peuvent pas suivre le clan durant la période de la chasse. Elles partagent leurs logements précaires avec les Indiens algonquins, ce qui favorise les rencontres et offre la possibilité d'associer les soins et le catéchuménat. Pour elles, cette expérience parmi les Indiens les renvoie à l'expérience de l'Église primitive vis-àvis des païens. Cette vision qui fait de l'Église missionnaire une reviviscence de l'Église apostolique, en expansion jusqu'aux limites du monde, rappelle l'ambition des empires coloniaux. À partir de l'analyse des Constitutions, du Directoire spirituel destiné aux novices, des Annales et d'autres documents, Catherine Fino permet aux lecteurs de comprendre l'expérience eschatologique des religieuses en tant que base de cette figure historique de la charité. Elle souligne le rôle actif des sujets (religieuses et indiens) qui par leur vie théologale et l'adhésion à la foi chrétienne - structurent et définissent l'espace hospitalier. Sans les analyser en détail, l'auteur mentionne aussi les options politiques, économiques et médicales qui contribuent à forger la pratique de la charité des Augustines de cette époque. L'assimilation politique des Indiens part du présupposé de la supériorité culturelle des Occidentaux. Le poids de la rationalité économique sur cette figure de la charité est illustré par le fait que c'est le financeur (la duchesse d'Aiguillon) qui impose le public de l'hôpital en privilégiant les Indiens. Au plan sanitaire, la prise en charge de la population française de la cité du Québec prend de l'ampleur. Les aspects politiques, économiques et médicaux permettent de saisir l'ambigüité, la fragilité et le déséquilibre grandissant de cette entreprise missionnaire.

5 Progressivement une nouvelle figure hospitalière se dessine. Plusieurs facteurs entrent en jeu: la diminution du nombre d'Indiens chez les patients, d'une part; le désengagement financier de la Duchesse d'Aiguillon, principale donatrice de la communauté des Augustines, d'autre part; et enfin le projet de construction d'un "Hôtel-Dieu à la française ", qui, davantage destiné aux Français, sera en droit d'attendre un soutien des autorités publiques.

6 Le troisième chapitre décrit l'implantation de l'Hôtel-Dieu de Québec sur une longue période jusqu'à la fin du Régime français en 1759. Dans un contexte colonial complexe, l'hôpital s'agrandit et se médicalise. Il accueille de nombreux marins et soldats; les religieuses travaillent de plus en plus aux côtés des médecins et des chirurgiens rémunérés par l'administration de la colonie puis par celle du roi. Dans ce chapitre, Catherine Fino s'intéresse à l'adaptation des religieuses face aux nouvelles exigences, et 
à la place de la charité dans ce nouveau projet. L'évangélisation des Indiens indissociablement liée à la première figure de charité est relayée par la nécessité de l'éducation morale et religieuse des pauvres. La moralisation des pratiques se traduit par la recherche de la sanctification des personnes qui vivent à l'hôpital (malades et soignants). Pour les Augustines, les comportements d'exception qui ont transgressé les normes habituelles de prudence pour un meilleur service des pauvres instituent une tradition locale. De ce fait, conclut l'auteur, la charité, capable d'induire et de consolider des pratiques inédites, acquiert aussi la capacité de transformer des institutions à long terme, au-delà de l'expérience individuelle de chaque sœur.

7 Finalement, le quatrième chapitre discute la thèse de l'extinction de la charité face à la médicalisation en analysant une figure hospitalière plus récente (fin $\mathrm{du}_{\mathrm{xix}}{ }^{\mathrm{e}}$ siècle) : l'asile Saint-Michel Archange. Dans cet asile, les sœurs de la Charité de Québec doivent seconder les médecins. Elles interviennent au titre du traitement moral et non médical des aliénés, ainsi que dans l'administration de l'établissement. L'auteur interroge alors les sources pour savoir si les nouvelles conditions des soins, marquées par l'essor de la psychiatrie, laissent aux religieuses la possibilité de vivre le service des malades comme un engagement de foi et de charité. Les hospitalières identifient l'asile comme un lieu privilégié pour exercer leur vocation et pour vivre une spiritualité qui confère aux épreuves politiques une dimension de renouvellement. La dimension sociale et politique de l'engagement des religieuses se concrétise dans la lutte pour la reconnaissance des diplômes d'infirmières et la professionnalisation du soin psychiatrique. L'analyse détaillée met en évidence le lien entre la formation reçue par les sœurs, la réalité institutionnelle et la mise en œuvre de la charité malgré la médicalisation croissante.

Dans sa conclusion, l'auteur souligne la dimension actuelle de son étude historique de la charité puisque celle-ci commande l'éthique des soignants. Elle lance alors un appel aux soignants croyants d'aujourd'hui et les invite à un travail d'imagination pour dessiner la figure de l'hospitalité dans un monde postmoderne pluriel. Cette exploration documentée et passionnante de l'histoire hospitalière veut convaincre les lecteurs que la charité est toujours un élément important de l'hôpital moderne qui ne peut s'en défaire, puisqu'il en est issu. Dans son ouvrage, l'auteur articule avec élégance engagement théologique et recherche historique. 